\title{
Desempleo y flexibilización: Alemania Federal y Chile frente a las transformaciones del capitalismo (Décadas de 1970 y 1980) ${ }^{1}$
}

\section{Unemployment and Flexibility: the German Federal Republic and Chile in the face of the transformations of capitalism (1970s and 1980s)}

\author{
Claudio llanos \\ Pontificia Universidad Católica de Valparaíso, Chile \\ José Antonio González \\ Universidad Católica del Norte, Chile \\ Joel Stillerman \\ Grand Valley State University, USA
}

\begin{abstract}
RESUMEN Este artículo analiza las respuestas a la crisis de los 1970s y la de 1982, de Chile y Alemania Federal. Crisis que impactaron en contextos diferentes, tanto a nivel de posiciones en la economía capitalista, como en lo referente a los regímenes políticos. En ambos países se plantearon el desempleo y los cambios en los ámbitos del trabajo. En este contexto, en Chile, las turbulencias económicas mundiales, se ligaron a la reacción violenta de los militares a la "vía chilena al socialismo" que, en alianza con las grandes empresas y economistas neoliberales, permitieron el desarrollo de las políticas neoliberales. Alemania Federal respondió al final del "milagro económico" de la posguerra con políticas gradualistas que preservaron el Estado del bienestar. La economía coordinada de Alemania Federal y la influencia de los sindicatos dentro de los dos grandes partidos políticos postergaron la aplicación de estas políticas hasta los 2000. La comparación demuestra la importancia de las configuraciones políticas de cada país para explicar sus políticas económicas y sociales frente a cambios de la economía global. De esta forma, se evidencia que la política es
\end{abstract}

1. Artículo parte del Proyecto Fondecyt Regular 1180074. 
el campo de construcción de las diversas alternativas frente a las dinámicas del capitalismo.

PALABRAS CLAVE Desempleo; flexibilización; crisis; Chile; Alemania.

ABSTRACT This paper analyzes Chile's and the Federal Republic of Germany's responses to the 1970s and 1982 economic crises. These crises affected these two countries differently, given their positions in the world economy and their distinct political regimes. In both countries, political debates focused on unemployment and the changing character of work. In this context, in Chile, the global economic crisis was the context for the military's violent reaction to the "Chilean road to socialism." The military alliance with big business and neoliberal economists facilitated the development of neoliberal policies. The Federal Republic of Germany responded to the end of the post-war "economic miracle" with gradualist policies that preserved the welfare state. The Federal Republic of Germany's coordinated market economy and the trade unions' influence within the two major political parties delayed the implementation of neoliberal policies until the 2000s. This comparison demonstrates the importance of each country's political configurations for explaining their economic and social policies in the context of a changing global economy. In this regard, politics is the key field for the development of diverse responses to global capitalist processes.

KEYWORDS Unemployment; flexibility; crisis; Chile; Germany.

\section{Introducción}

Durante los años 1970 y 1980 la economía mundial enfrentó un conjunto de problemas y transformaciones. El término de los acuerdos de Bretton Woods (1971); las dos crisis del petróleo (1973 y 1979) y la crisis de la deuda (1982), fueron acontecimientos que impactaron con diversa intensidad a los países, generando importantes discusiones políticas relacionadas con las medidas necesarias para enfrentar las nuevas realidades económicas. Aún con las divergencias en los impactos, es posible observar fenómenos generales comunes en el ámbito del trabajo, y dentro de estos el desempleo y la flexibilización asumieron características relevantes.

En este artículo pesquisamos las dinámicas e intensidades que dichas transformaciones asumieron en Chile y Alemania Federal. Estos fenómenos están insertos en procesos histórico globales, que se han desplegado en América Latina, a distintos ritmos e intensidades, configurando realidades nacionales, que no se pueden entender fuera de sus relaciones con las dinámicas del capitalismo, sus transformaciones y sus impactos en las condiciones y formas de trabajo, incluidos los temas de la seguridad laboral y trabajo femenino. 
El análisis de los dos países se funda en las formas en que los fenómenos internacionales paralelos, se enfrentaron en sociedades con regímenes políticos diferentes, en Chile una dictadura que había clausurado la discusión política y democrática, que llevó adelante un conjunto de modificaciones en lo político, social y económico, sin contrapesos. En contraste, Alemania Federal debió enfrentar procesos de crisis y cambio, en los marcos de una democracia multipartidaria, con importante participación de los sindicatos en las discusiones y decisiones políticas.

En este marco, las reformas, que se comenzaron a aplicar desde la temporalidad en estudio, dependieron en gran medida del régimen político, donde a mayor debate y posibilidades de participación, se dieron más limitaciones o intensidades menores de las políticas de desregulación y flexibilización laboral.

Empero, la OIT hacia fines de la década de los 90, consideró que la flexibilidad posibilitó aumentar la productividad y los niveles de empleo, en determinados sectores vinculadas a las innovaciones tecnológicas -industrias eléctricas, electrónicas y mecánicas- pero enfrentó viejas prácticas laborales y fundamentalmente desafíos para determinar cómo implementar tales prácticas de gestión, lo que se reflejó en afectar al $15 \%$ de los trabajadores alemanes. La flexibilidad acrecentó el empleo a nivel mundial en la década del 80 en un 12\% (OIT, 1998).

Los cambios que ha experimentado el mundo del trabajo desde los años 1980 han estado crecientemente marcados por la tensión entre medidas autoritarias, participación democrática y la importancia de los trabajadores y sus organizaciones.

Los ejemplos de Chile y Alemania Federal, representan dos modelos de aplicación de la flexibilidad en dos regiones diferenciadas - un país céntrico y otro semi-periférico del sistema capitalista mundial - donde, además de las diferencias de regímenes políticos, que muestran consideraciones socio-políticas diversas, se encuentra la influencia de los sindicatos- en el caso alemán- que incidieron en los partidos políticos hegemónicos para incentivar políticas de ajuste gradualistas, en contraposición con la situación chilena, donde estas consideraciones estuvieron ausentes, y se aplicó como herramienta de potenciar el mundo empresarial en desmedro de los trabajadores, castrados de representación sindical legítima.

Considerando la importancia de las discusiones y reformas en torno al desempleo y la flexibilización, la temporalidad estudiada constituye un tiempo eje para aproximarnos a los diferentes desarrollos y cristalizaciones, que dieron forma a realidades y problemas sociales que continúan manteniendo su relevancia y actualidad, como fenómenos histórico sociales. Estas transformaciones han afectado a los trabajadores y trabajadoras, sus derechos, condiciones y relaciones, y constituyen temas que se mueven más allá de temporalidades o territorios rígidos. Nuestra investigación, persigue contribuir al conocimiento del impacto de las turbulencias globales, en sociedades que aunque distantes y diferentes, son parte del sistema capitalista. 
Durante las crisis de las décadas de 1970 y 1980, en un número importante de sociedades capitalistas, se planteó el aumento en el desempleo. Este fenómeno afectó a diversos países centrales y semi-periféricos del capitalismo. Las políticas de bienestar y desarrollo generadas desde las décadas de 1930 y 1960 fueron cuestionadas, entre otras, por las ideas y propuestas neoliberales.

El alto desempleo fue uno de los elementos de fricción entre las políticas que habían considerado que la meta del empleo pleno era una función del Estado, y el neoliberalismo que persigue desplazar la acción económica y política, lejos de las ideas de bienestar, igualitarismo y justicia social, para centrarse en el manejo monetario y el control de la inflación.

Las crisis de 1970 y de 1982, permitieron una arremetida del neoliberalismo, que logró avanzar con sus propuestas de ajuste económico, cambios y reducción de políticas y derechos sociales. En los últimos años la historiografía y las ciencias sociales en general, han puesto creciente atención a las décadas de 1970 y 1980 como momento de "rupturas" relevantes en las sociedades.

En contextos de crisis económica y aumentos en el desempleo, se desplegaron discusiones orientadas a reducir las "rigideces" del mercado laboral, y la necesidad de flexibilidad en el empleo, como formas de enfrentar los problemas generados por el ascenso de nuevos actores productivos, los cambios en los procesos de industrialización internacional, incluido el método "Just in time" que implicó el agotamiento del fordismo.

La flexibilización laboral se configuró como un fenómeno global, con distintas intensidades de implementación que comenzaron a desplegarse desde la segunda mitad de los años 1970, pero que fueron tomando impulso y cristalización política desde los años 1980 en adelante. Esto nos permite entender que aun con distintos momentos de implementación en Chile y Alemania Federal, los procesos de flexibilización laboral son parte de procesos de transformación global.

La importancia de dirigir una mirada a ambas trayectorias, está en que experiencias de estos países y sus sociedades, nos aproximan a las dinámicas y cambios que el capitalismo ha imprimido en las formas de trabajo. La historia de la flexibilización del trabajo, constituye una historia común para sociedades diversas.

En lo que corresponde a lo central y semi-periférico, nos concentramos en las discusiones políticas en Alemania Federal y Chile, durante los años señalados ${ }^{2}$. Con ello además de pesquisar las intensidades del discurso y las prácticas neoliberales, se iden-

2. En el plano específico del estudio comparado de discusiones políticas, una de las mayores dificultades, para la investigación en ámbitos de los procesos políticos, económicos y sociales del capitalismo y sus "variedades" está en el acceso y disponibilidad diferencial de fuentes e información, para las diversas experiencias históricas. Además, en términos de la investigación histórica, el tema del desempleo ha recibido mayor atención en Alemania en comparación a Chile. En este último caso podemos encontrar desde la historia trabajos de Ángela Vergara y otros que si bien cubren la historia del trabajo se concentran más en la relación con los temas sindicales y organizativos. 
tificarán tres formas en las cuales se diferencian estos países. Primero, es evidente que la dictadura en Chile dificulta las posibilidades que grupos organizados en la sociedad pudiesen contrarrestar las políticas neoliberales desarrolladas por el gobierno militar. $\mathrm{Al}$ contrario, las políticas de ajuste que se implementaron en Alemania se realizaron bajo un gobierno democrático que contó con instituciones de negociación colectiva y desarrollo de políticas económicas "corporativistas" que ponían el enfoque en la confección consensuada de políticas de mercado de trabajo y de bienestar entre grupos organizados (sindicatos, organizaciones empresariales, y el Estado).

Segundo, estos dos casos ocupan lugares distintos dentro de sus respectivas regiones. En contraste con los otros gobiernos militares de Sudamérica durante el período (Brasil, Argentina, y Uruguay), Chile aplicó políticas neoliberales de una forma sostenida. Los otros países realizaron experimentos neoliberales de corta duración y limitados efectos (Argentina y Uruguay) o desarrollaron políticas que profundizaron la substitución de las importaciones (Brasil).

En Chile, desde los años 50 hubo una formación de economistas neoliberales, que afianzaron un programa de gobierno, con entidades productivas y sectores financieros, que convergieron en dictadura (Fischer, 2009, p. 330; Fontaine, 1988; Vergara, 1984). La experiencia chilena sirvió de lecciones para aplicaciones en regímenes democráticos, países anglosajones, y a Argentina, en los años 80 y 90 (Muiños, 1999). Es la radicalidad del proceso chileno, lo que orientó sobre las posibilidades, limitaciones y mecanismos de implementación neoliberal en condiciones menos "ideales".

El caso alemán ocupa un punto intermedio entre los países del libre mercado (Estados Unidos e Inglaterra, entre otros) y los países social-demócratas (Escandinavia). Con su herencia de un Estado de bienestar conservador de la época de Bismarck e instituciones corporativistas de negociación colectiva, Alemania logró proteger el empleo industrial masculino con más éxito que los países liberales, pero desfavoreció el empleo femenino y juvenil. En contraste, los estados social-demócratas ofrecieron servicios gratis de sala cuna y promovieron el empleo público, dos políticas que tuvieron efectos más igualitarios para distintas categorías de trabajadores y para los hombres y mujeres (Thelen, 2014).

Tercero, las diferencias estructurales en lo económico y políticas entre Alemania Federal y Chile, radican en tres aspectos importantes con relación al fenómeno del desempleo y los cambios en la regulación laboral en el horizonte de la flexibilización del trabajo. El impacto de la crisis de la deuda latinoamericana (1982-1985), a causa, entre otros factores, al alza de la tasa de interés de la Reserva Federal de los EE.UU., en 1981, golpeó fuertemente al sistema financiero chileno. Alemania, un país acreedor, vio disminuida la rentabilidad del capital en un 8,8\% entre 1970-1975 golpeado por la crisis del dólar en 1971 y los precios del petróleo en 1973 (Rapoporty Brenta, 2010). Además, Alemania Federal mantuvo la economía social de mercado, donde 
el consenso y la cooperación, equilibró en su sistema productivo al sector financiero como predominante. En Chile, el renovado sector financiero, fue impulsado por la política neoliberal monetarista, que clausuró el proceso de industrialización/empleo, y situó al sector inmobiliario como un nuevo actor (Daher, 2013), que se tradujo en una profundización de la crisis social, con la segregación espacial, y promovió un aumento del empleo informal durante los años 80. Por último, Chile fue un país exportador de materias primas que desarrolló un sector industrial bajo el alero del Estado con el objetivo de satisfacer el mercado interno. En contraste, Alemania tuvo una historia más larga de desarrollo de industria pesada que producía productos de calidad con altos niveles de exportación (Streeck, 1997).

A partir de estas diferencias de historia política, políticas sociales, y estructuras económicas, planteamos que los dos países experimentaron la crisis global de los 70 y 80 de una forma diferente, y a su vez, respondieron a esa crisis de una forma distinta. En Chile, el gobierno militar respondió a una crisis socio-política que coincidió con problemas de larga duración como la inflación y limitaciones del desarrollo industrial. Con el apoyo de sectores de derecha, economistas neoliberales, y grandes empresas, los militares lograron imponer políticas neoliberales sin tener que negociarlas con la ciudadanía. Estas políticas significaron una transformación de la estructura de propiedad y las políticas sociales además del aumento de la pobreza, trabajo precario femenino e informalidad. En Alemania, el sistema de economía coordinada bajo un gobierno democrático conjugo con el poder de los sindicatos dentro del partido socialdemócrata y la democracia cristiana para incentivar políticas de ajuste gradualistas que protegieron el empleo industrial masculino. Sin embargo, estas políticas limitaron el acceso a empleo de las mujeres y los jóvenes, en contraste con los gobiernos escandinavos que liberalizaron sus mercados laborales mientras aumentaron sus sistemas de beneficios sociales. En los dos casos, los contextos políticos y de alianzas permitieron distintas respuestas a la crisis económica global con diferentes consecuencias para el trabajo y la desigualdad económica.

\section{Crisis de los años 1970 y 1980}

Los cambios económicos internacionales que arrancan desde la década de 1970, con dos crisis del petróleo incluidas, plantearon al conjunto de las sociedades capitalistas, una importante modificación en sus puntos de referencia en cuanto a los significados de la estabilidad económica y su relación con la sociedad. Se comenzaron a discutir un conjunto de reformas a las relaciones laborales, que cristalizaron politicamente desde la década de 1980 en adelante.

En los países industrializados, desde el comienzo de la Gran Depresión hasta los años 70, el Estado jugó un rol central en el desarrollo de políticas económicas que integraron las demandas de sindicatos y otros sectores económicos en las medidas 
fiscales, redistributivas y de planificación económica. En efecto, parte importante del siglo XX fue un tiempo de predominio del Estado (Sassoon, 2001).

Después de 30 años de crecimiento económico entre aproximadamente 1945 y 1975, con alto empleo y producción, se planteó "el fin de la sociedad industrial", lo que no solamente se refiere a las modificaciones dentro del capitalismo internacional y su división del trabajo, con crecientes masas obreras industriales fuera de las metrópolis, sino que también involucró cambios a nivel de la vida social, la familia, la cultura, etc ${ }^{3}$.

Al final de este período, el aumento en el número de desempleados significó una fuerte presión, tanto para las políticas de bienestar avanzadas de los países céntricos, como para aquellas desarrolladas en las economías semi-periféricas. Entre los años 1960 y 1989, los países de la OCDE (de los EEUU, Europa y Japón), el componente de indemnización por desempleo (como porcentaje dentro del gasto social) pasó en promedio desde 2,9 en 1960 a 5,5 en 1985 (Torres y González, 1993, p. 289). Estos aumentos se dieron, en un marco general donde el Gasto Social en la mayoría de los países europeos, "se ha ampliado desde 1960, a pesar de que el ritmo de crecimiento se ha disminuido desde 1990" (OCDE, 2019, p. 2).

En lo que corresponde a los aumentos del gasto y presiones económicas debidos a los aumentos del costo de petróleo, "El modelo de crecimiento de la postguerra y la teoría de la expansión de la demanda agregada que inspiró la actividad económica del sector público quedaron agotados en 1973. Entre este año y 1982 la profunda crisis económica afectó decisivamente tanto a la intervención del Estado en la economía como a los mecanismos con los que hasta entonces la había legitimado" (Torres y González, 1993, p. 281).

La década de 1970 constituyó el término de períodos de expansión de los Estados de Bienestar en las sociedades capitalistas occidentales (1945-50, 1950-6o y 1960-73), y el ingreso en períodos de ajuste y reducción de las políticas de bienestar (retrenchment) en los siguientes "subperiodos" identificables en "1973-9, 1980-89 y 1990 a hoy" (Nullmeier y Kaufman, 2010, p. 87).

En este horizonte, desde la segunda mitad de los años 1970, las políticas económicas en las sociedades capitalistas occidentales estuvieron marcadas por las lógicas del ajuste económico, y en lo que corresponde al trabajo se perfilaron fenómenos y políticas orientadas a la flexibilización laboral, en un marco internacional de externalización productiva (Lessenich, 2017).

3. El cuestionamiento a la fidelidad al modelo socialdemócrata por la nueva clase obrera en cuanto a los criterios de una "sociedad de consumo" conjuntamente con los cambios generacionales, los jóvenes más vuelto hacia la subjetividad y las nuevas utopías, posibilitaron los argumentos neoliberales del agotamiento de la oferta socio-económica del Estado de bienestar (Offe, 1990). 
El periodo abierto en los años 1970, representó un tiempo de fuertes presiones sobre los Estados de Compromiso de América Latina, que desplegados desde los años 1930 y 1940 habían logrado balancear los desarrollos del capitalismo y las demandas de importantes sectores de los trabajadores organizados. Presiones que se relacionaban tanto con el aumento de gasto público y social, como con críticas a su funcionamiento. Por cierto, los cambios que experimentaron los Estados de Compromiso, fueron de diversa intensidad, en relación a las características de las políticas que se implementaron desde los años 1970 y 1980.

En ese escenario, parte importante de la crítica de economistas neoliberales apuntó contra la importancia que habían asumido las organizaciones de trabajadores, dentro de las dinámicas económicas. Hayek y Friedman, entre otros, les presentaron como generadoras de alteraciones económicas, como la inflación, debido a los aumentos salariales, y que desde esa perspectiva argumentan que los trabajadores mejor organizados afectan a los sectores productivos más débiles, dañando su capacidad de consumo, y al mismo tiempo generando incentivos al reemplazo de mano de obra "humana" por la tecnología".

\section{Transformaciones centrales y semi-periféricas y la importancia del régimen po- lítico y sistemas de coordinación económica: los casos de Alemania Federal y Chile}

En Alemania Federal, las crisis económicas inauguradas en la década de 1970 significaron el término de lo que desde los años 1950 se conoció como el "milagro económico" alemán (Wirtschaftswunder). El aumento en el costo de la energía, ligado al aumento de la competencia internacional en la producción industrial, implicó variados fenómenos, como caídas en la producción que afectaron al empleo de algunos sectores, con ejemplos en la industria textil, que entre 1974 y 1975 perdió un 10\% de

\footnotetext{
4. En un plano general, la pugna entre tecnología (capital) y trabajo se presentó como una resultante de los elevados costos de mano de obra, en un escenario donde desde los años 1960/1970 se observa tanto el agotamiento del impulso económico de postguerra, la ampliación del número de países que participaban en la producción industrial mundial, es decir el puñado de potencias capitalistas occidentales, perdida de terreno frente al avance de la producción industrial asiática, desde inicios de los años 1980, además de un incremento de las desigualdades a nivel internacional y nacional. Sobre esto ver (Piketty, 2014, p. 75). En el caso específico de Alemania Federal, si bien la pérdida de su posición internacional no fue tan pronunciada en los años 1970, el impacto de las convulsiones económicas de los años 1970 y 1980 generó problemas en la industria textil, acero y minería, con aparición del desempleo masivo y el debate sobre "el fin del trabajo", (Süß y Woyke, 2012, p. 4). Sobre esto también ver (Beaud, 2012, pp. 303-308, 318-321). Los fenómenos económicos experimentados desde los años 1970 se vincularon a procesos de transformación tecnológica en la producción y las comunicaciones que marcaron un tipo de "globalización", donde entre otros temas "se incrementaron las opciones de salida de capital (financieras); la posibilidad de búsqueda mundial por las locaciones más competitivas para inversión" (Nullmeier y Kaufman, 2010, p. 93).
} 
los puestos de trabajo (Lindner, 2008, p. 60). En la industria de automóviles, la década de 1970 planteó la presión de costos y competencia, que le llevaron a la innovación tecnológica, donde la robotización fue un elemento central de una racionalización flexible de producción, que impactó directamente en el número de trabajadores de esa industria.

En noviembre de 1973, dentro del círculo del canciller se escuchaba de la "violenta tormenta" (Heftiger Sturm) que afectaría la llegada de petróleo (Der Spiegel, 1973, p. 25). Este no era solamente un problema climático en Medio Oriente, que podría afectar a la llegada de petróleo, sino que efectivamente se produciría un golpe en la situación económica. De esto el canciller Willy Brandt era consciente, tal como lo señaló ante el Bundestag el 29 de noviembre de 1973 (Deutscher Bundestag, 1973, p. 3908).

A finales de 1975, el número de empleados en las plantas de fabricación final de automóviles retrocedió en cerca del 13,5\% (Bauer, 2008, p. 73). Sin embargo, en términos comparados, en esta época el movimiento sindical alemán logró minimizar los efectos sociales de estos cambios a través de una estrategia de negociar aumentos salariales moderados, reducir las horas trabajadas por semana, y reducir la fuerza laboral a través de las jubilaciones en vez que los despidos. Además, las instituciones de desarrollo industrial y negociación colectiva consensuadas, combinadas con su sistema de aprendizaje para aumentar la calificación de los obreros permitieron que las empresas industriales más intensivas en capital pudieran competir en el mercado mundial a través de la calidad de sus productos en vez de su bajo precio. Este modelo de "calidad diversificada de productos" (diversified product quality) durante los años 70 y 80 logró minimizar las diferencias entre los salarios de los distintos grupos de trabajadores, incentivar la capacidad competitiva de las empresas alemanas, y disminuir los efectos negativos de la cesantía en los trabajadores (Baccaro y Benassi, 2017, p. 95; Hall y Thelen, 2009, pp. 16-19; Kenworthy, 2002, pp. 369, 384; Möller, 2015, pp. 152-156; Streeck, 1997, pp. 238-245; Thelen, 2012, pp. 140, 143-145).

En Chile, la crisis de inicios de los años 1970, impactó a una sociedad y economía en crisis. Empero, una crisis larvada, en materias de empleo, seguridad social y crecimiento económico, y visible, en las esferas político-social, por la mayor injerencia del Estado en fijar los salarios en la actividad privada, el control de empresas importantes, vinculadas con la Corfo, y un aumento del tamaño del Estado equivalente al 10,1\% del PIB en la década de $1960^{5}$. Dentro de las paradojas y signos de la nueva política en la dictadura, encontramos simultáneamente, la reducción del tamaño del Estado a un 5\% del PIB y la liquidación de los subsidios estatales (favo-

5. Klaus Schmidt- Hebbel, "El crecimiento económico de Chile". Banco Central de Chile. Documento de Trabajo $\mathrm{N}^{\circ} 365$, junio 2006, p.6. El crecimiento del PIB era de 1,5\% anual. 
reciendo el incremento de las reservas internacionales), y el rescate entre 1982-83 y 1985-1988 de la quiebra de la banca privada, invirtiendo en el sector privado el equivalente al 102,7\% del PIB (Fillipi, Román y Villena 2017).

La dictadura militar, y sus políticas económicas, afectó a la población, con acciones directas e indirectas en la vida cotidiana chilena. En las directas, afectó el empleo durante la primera fase de privatización entre 1973-1974, con la devolución a sus dueños de los fundos expropiados y las 325 empresas intervenidas por la Unidad Popular, y la enajenación de empresas estatales asociadas a la Corfo (Hachette, 2000, pp. 114-116). Posteriormente, en la segunda fase de privatizaciones, de 1975 a 1983, se privatizaron 207 empresas intervenidas. Las indirectas, que afectaron a la población fueron evidentes, como las transformaciones estructurales de la economía y la paralización del sindicalismo (Drake, 2003, p. 150).

La dictadura militar, experimentó desde 1974-1975 crecientes niveles de desempleo, que rompieron la tasa cercana al 5\% entre 1960-1973, observándose una alza sostenida hasta llegar en 1982 al 21,8 \%. El fracaso de la política de reducción del desempleo hizo que entre 1975 y 1983 los niveles que se empinaran a cerca del 15\% y $20 \%$. Si se incluye la población inscrita en el Programa de Empleo Mínimo (PEM) y Programa de Ocupación para Jefes de Hogar (POJH), la cesantía efectiva se acercó al 30\% de la población económicamente activa en 1983 durante la crisis financiera (Llanos, 2018; Riveros, 1984, p. 5; Winn, 2004, p. 41).

Desde 1975, plantas industriales como las armadurías de autos (Ministerio del Trabajo, Chile, 1975), experimentaron contracciones que marcaron su progresivo debacle durante los años 1980 y 1990. Sin embargo, después de la crisis financiera de 1982-1983, el empleo industrial se recuperó, pero este incremento se dio en los nuevos sectores de exportación como la agroindustria, la industria pesquera, y la industria forestal, mientras hubo una contracción de empleo en los sectores tradicionales de la industria como las empresas textiles y metalúrgicos (Martínez y Díaz, 1996, pp. 103-107).

Las economías capitalistas, centrales y semi-periféricas, vieron el aumento del desempleo, ligado a incrementos en costos productivos y a transformaciones tecnológicas que impactaron en la relación capital-trabajo. Para el horizonte del trabajo y el empleo las diferencias se pueden explicar a nivel de las políticas y decisiones tomadas por los Estados y el grado de influencia y capacidad de las críticas lanzadas contra la centralidad del empleo en las políticas públicas. Estos grados de penetración y capacidad de implementación estaban en relación con la existencia o no del régimen democrático y la herencia de instituciones de negociación colectiva y de Estados de bienestar.

De esta forma, las reformas que afectaron la importancia del empleo, frente a otras consideraciones económicas (por ejemplo, la inflación) dependieron en parte 
de la situación política de cada país. En el caso del neoliberalismo, en los dos casos considerados, la democracia resultaba una limitante, inexistente en Chile, lo cual explica en parte la temprana aplicación de sus propuestas.

La posibilidad de la discusión democrática y la movilización social y política, permite entender tanto la intensidad de implementación de las críticas de los economistas neoliberales, con sus argumentaciones y propuestas políticas que perseguían terminar con el Estado de Compromiso y la acción económica del Estado, como los fracasos o límites que dicha agenda ideológica experimentó.

Es importante contextualizar este punto en dos aspectos. Primero, Chile fue el pionero en aplicar las políticas neoliberales de forma sostenida en la región latinoamericana y el mundo. Las dictaduras contemporáneas de Brasil, Uruguay, y Argentina no implementaron estas políticas. Los militares brasileños optaron por profundizar la política desarrollista de sustitución de las importaciones y los militares de Argentina y Uruguay implementaron políticas neoliberales por periodos cortos y en muchos casos miembros de la Junta del gobierno vetaron estas políticas. En contraste, la presencia de economistas neoliberales formados en la Universidad de Chicago antes del gobierno de Allende con apoyo de algunos empresarios, sectores de la derecha política, y militares de la Marina fue una precondición para el experimento neoliberal. De hecho, el programa de intercambio entre la Universidad Católica y la Universidad de Chicago (que fue parte de la política exterior de los Estados Unidos que quiso contrarrestar la influencia de ideas de izquierda entre los economistas en América Latina) fue replicado en otros países como Argentina y Colombia durante los 50 y 60, pero no tuvo el mismo éxito en influir las políticas económicas en aquellos países que en Chile. Además, el gobierno personalista de Pinochet que logró marginar competidores más desarrollistas dentro de la junta permitió la aplicación de políticas neoliberales sin respuesta dentro o fuera del gobierno. En fin, durante los años 70 en América Latina, la existencia de un gobierno militar fue necesario, pero no suficiente para explicar la aplicación sostenida de las políticas neoliberales en Chile (Biglaiser, 1999, pp. 1520; Drake, 1996, pp. 76-90; Fischer, 2009, pp. 308-311; Fourcade-Gourinchas y Babb, 2002, p. 547; Huneeus, 2001, pp. 389-414; Winn, 2004, pp. 19-29).

Segundo, después de la implementación de las políticas neoliberales en Chile, otros gobiernos no-dictatoriales implementaron políticas similares. Curiosamente, el caso más similar al chileno en relación a la profundidad de sus reformas neoliberales es Inglaterra, que los implementó unos pocos años después bajo un gobierno democrático, pero sin las masivas violaciones de derechos humanos que se dieron en Chile. Una explicación posible de las similaridades entre estos dos casos es que los grupos neoliberales llegaron al poder como reacción a intensos conflictos sindicales y períodos de alta inflación en comparación con otros países similares. El caso inglés contrasta con los casos de economías coordinadas de mercado como los países nór- 
dicos o Alemania donde las altas tasas de sindicalización y la posibilidad de negociar con empresarios organizados y el Estado lograron limitar las huelgas y controlar el aumento de salarios. Es importante entonces ver a Chile como un "laboratorio" cuyos resultados y "errores iniciales" sirvieron como insumos para políticas en otros países y de las instituciones multilaterales. Por ejemplo, el FMI y el Banco Mundial, usaron elementos del modelo chileno en el manejo de la crisis de la deuda de los 80 (Fischer, 2009, pp. 332-333; Fourcade-Gourinchas y Babb, 2002, pp. 538-539, 549-556; Harvey, 2005, pp. 9, 39, 63, 115).

Un rasgo y proceso común entre los dos países, fue la progresiva expansión de la flexibilización laboral. Esta implicó desde los años 1970 "una nueva sincronización del tiempo de trabajo y el tiempo libre, donde el discurso económico capitalista en un marco de alta competitividad señalaba la necesidad de nuevos individuos capaces de combinar el trabajo y su vida" (Süß, 2012, p. 142). Dichos procesos estaban ligados a las discusiones sobre el tiempo de trabajo, el desempleo, y racionalizaciones de la producción "postfordistas" que se desarrollaban a partir de los cambios que la tecnología generaba dentro del trabajo y la cadena productiva ${ }^{6}$.

La flexibilización laboral planteó, por lo menos dos importantes dimensiones: primero su relación con el trabajo "femenino" y segundo el surgimiento del subcontrato, o contrato atípico, fenómenos que fueron presentados como mecanismos para elevar la participación en el empleo en contextos cada vez más inciertos y competitivos. Además de caracterizarse por una reducción de las "seguridades" laborales, en favor de las necesidades de los empleadores (del capital).

En Alemania, intelectuales feministas plantearon la meta de terminar con el deber de la mujer en el trabajo doméstico, insertándose además dentro de la demanda salarial por esta actividad (Kramer, 2012, pp. 213-214). Las posiciones del feminismo ampliaron el debate en torno a la participación de las mujeres en el trabajo asalariado, la flexibilidad de éste, la expansión de la educación para las mujeres, las implicancias y significados del trabajo reproductivo en la identidad y la inclusión social.

En el plano general europeo, se observó una creciente participación de las mujeres en el trabajo remunerado que pasó de 33\% en 1950 a 36\% en 1970 (Kramer, 2012, p. 213; Schwartz, 2008, pp. 196-198). Según Blossfeld et al. (2008), este fenómeno estuvo marcado por una expansión educativa y de la formación profesional de las mujeres desde los años 1960. Acompañado de mejoras en las condiciones para desarrollar carreras profesionales más exitosas en relación a mujeres de generaciones anteriores. Aún con el aumento de la participación de las mujeres en el empleo remunerado, "en

6. Expresión política de las nuevas dinámicas temporales es el debate y reducción de las horas semanales de trabajo que en Alemania Federal pasaron de 45,6 horas semanales los años 1960; a 44,1 en los 1970 y a las 41,6 en los años 1980 (Ver Süß, 2012, p. 143). 
todas las sociedades modernas, las tareas de cuidados familiares son no remuneradas y están ocupadas exclusivamente por mujeres" (Blossfeld et al., 2008, p. 32).

Desde los años 1970, el despliegue de la flexibilización laboral, en el caso de la participación de las mujeres se relaciona con las formas de regímenes políticos, por lo cual es relevante la relación entre capitalismo, trabajo y condiciones políticas que desde el Estado se establecen para las relaciones productivas. En este sentido los modelos políticos, socialdemócrata, conservador y liberal ofrecen distintas posibilidades de integración de la mujer a la vida laboral remunerada. En los socialdemócratas, se ofrecen mejores "servicios públicos y privados y la promoción de medidas favorables a la familia", junto a "buenas y estables oportunidades de empleo a las mujeres". En los modelos conservadores, (por ejemplo Alemania, Italia y España), se presenta una escasa "infraestructura de atención infantil", lo cual dificulta la integración laboral, planteando los cuidados domésticos (no remunerados) como un tema femenino. Los países liberales (por ejemplo los Estados Unidos de Norteamérica) ofrecen poco apoyo a familias, y la inserción laboral está en los márgenes del libre mercado, con lo cual muchas mujeres participan dentro del sector informal, con bajos salarios y seguridades sociales, esto particularmente en el caso de las familias de ingresos reducidos (Blossfeld, 2008, pp. 36-37).

En Alemania Federal, la flexibilización del trabajo se vio ligada al crecimiento del trabajo a tiempo parcial, que experimentó un crecimiento de 6,3\% en 1970 al 17,2\% en 1991 (Spitznagel y Wanger, 2004, p. 3). Desde fines de los 70 y durante los 80, las demandas sindicales plantearon la reducción en las horas de trabajo como forma de enfrentar el desempleo, reducción que se detuvo en los años 90 pero el empresariado siguió adelante con la flexibilización, en los marcos de una "desnaturalización de la relación laboral normal" y de "las normas del derecho laboral y de los convenios colectivos" (Hielscher, 2000, p. 14).

En el caso específico de la participación de las mujeres en los trabajos "parciales" se observa en Alemania el crecimiento de los "contratos atípicos" y de los "mini-jobs" particularmente desde la década de 1980, en el marco de una "crisis de las relaciones normales del trabajo" (Krise des Normalarbeitsverhältnisses)".

Este fenómeno experimentó los mayores incrementos desde inicios de los años 1980 y en lo que se refiere al trabajo parcial cualificado, para Heidi y Frank Oschmiansky "El empleo a tiempo parcial cualificado aumentó durante todo el período.

\footnotetext{
7. Esta crisis implica, "La pérdida de importancia de la relación laboral estándar. También se asocia con el temor de que el poder integrador del mercado laboral disminuya y que cada vez más gente tendrá, si es que tiene, una reducción significativa de participación en la seguridad social y las oportunidades de desarrollo social. El aumento de las formas atípicas de empleo podría conducir a una segmentación permanente del mercado laboral, donde parte de la fuerza de trabajo permanece permanentemente en relaciones laborales inestables" (Oschmiansky y Oschmiansky, 2003, p. 1).
} 
La expansión de esta forma de empleo de un 10\% a un 15,6\% entre 1985 y 2001 ha contribuido en gran medida a la creciente participación de las mujeres en el territorio federal" (Oschmiansky y Oschmiansky, 2003, pp. 3-24). La falta de acceso a trabajos de tiempo completo de las mujeres y jóvenes está relacionada con el diseño del Estado de bienestar en Alemania. El "modelo alemán” partió con el supuesto del "hombre proveedor" y por tanto el sistema de jubilaciones está ligado a los aportes de los trabajadores. Como el Estado no provee servicios gratis de sala cuna para toda la población, inhibe la participación laboral de las mujeres y desincentiva la fecundidad. Además, en contraste con los países nórdicos, el gobierno alemán no ha optado por crear empleos públicos para la población femenina y juvenil que no logra conseguir trabajos estables. Los costos del sistema de jubilación y seguro de cesantía subieron para los empleadores, y, por tanto, han dificultado la generación de nuevos empleos. Tanto el diseño del Estado de bienestar, las políticas del empleo de los gobiernos, y el sistema de relaciones industriales crearon un mercado laboral segmentado dividido entre hombres con empleo estable y mujeres y jóvenes que están más vulnerables a la cesantía o relegados a trabajos precarios. No fue hasta las reformas Hartz de los años 2000 que el gobierno logró enfrentar este problema, al dar posibilidad de generar empleos de tiempo completo con menor remuneración (Esping-Anderson, 1996, pp. 72-84; Möller, 2015, pp. 160-165; Thelen, 2012, pp. 152-154).

El gobierno demócrata cristiano de Kohl y su propuesta de "cambio" y "viraje" (Die Wende) no logró reducir los índices de desempleo en Alemania Federal, que desde 1983 hasta la unificación (1989-1990) estuvo en un promedio cercano al 8\%, y al momento de la unificación alcanzaba a casi dos millones de alemanes (occidentales). Así, los años 1970 y 1980 significaron una fuerte ruptura de los índices de desempleo de los años 1960, con promedios que se movieron entre el 1,1 (1960) y 0,9 (1969), con un máximo de $1,5(1968) \%^{8}$.

Las propuestas de transformación de Kohl y su relación con un "momento neoliberal" son un aspecto de discusión. Lo que sí es claro, es que los niveles de discusión política en un régimen democrático con un sistema institucional de una economía de mercado coordinada limitaron muchas de las propuestas cercanas al horizonte neoliberal en Alemania Federal. Aspectos de la política de orden keynesiano dieron paso a orientaciones neoliberales desde mediados de los años 1970 (Werding, 2008, pp. 303-321). En el contexto de estas discusiones, Helmut Kohl intentó implementar políticas neoliberales entre 1982 y 1987. Sin embargo, el ala obrera de su partido bloqueó esas iniciativas por considerarlas contrarias a sus principios y los intereses de sus seguidores. La estructura del Partido Demócrata Cristiano implicó que, su sector

8. Fuente: Layard, Nickell y Jackman (2003, pp. 526-528). 
obrero mantuviera su influencia sobre las políticas sociales y laborales, con lo cual ese sector fue un importante opositor a posiciones neoliberales (Prasad, 2006, pp. 167-192, 224-232).

En Alemania, se desarrollaron discusiones sobre la "nueva cuestión social" y la "nueva pobreza", particularmente en el caso de los jóvenes y el trabajo femenino no remunerado y las diferencias salariales frente a los hombres (Kramer, 2012, pp. 213 - 219). En este sentido, es importante tener en consideración los límites de estas definiciones sobre lo "nuevo", pues como ha señalado Ulrich Beck y Elizabeth BeckGernscheim, la modernización de la sociedad no solo implica nuevos riesgos y opciones, sino que también la permanencia de las viejas estructuras de la desigualdad, pues las relaciones desiguales de poder entre los grupos sociales no cambiaron profundamente sino se operó un cambio en los significados sociales de dicha desigualdad (Beck y Beck, 1994).

Las medidas neoliberales aplicadas en Chile, conjugaron una doble flexibilización: la primera afectando directamente al trabajo, de modo gradual, cuestionando las disposiciones establecidas en el Código del Trabajo de 1931; la disposición de la inamovilidad laboral de 1966, con normativas que desde 1978 posibilitaron los despidos de trabajadores sin especificación de causa; la resistencia al aumento del salario mínimo, que podría afectar negativamente al empleo, siendo modificado en el marco del Plan Laboral de 1979 (Rosende, 1988, pp. 67-128), y la segunda replanteando el criterio de seguridad social, que se había mantenido incluso en el gobierno tecnocrático de Alessandri Rodríguez (1958-1964), como lo había planteado el Informe de Jorge Prat Echaurren en 1964, al indicar que la seguridad social era la política "por la cual la comunidad protege a sus miembros asegurándoles condiciones de vida, salud y trabajo, socialmente suficientes, a fin de lograr mejor productividad, mayor progreso y más bienestar común", abriendo las opciones de salud y previsión en el ámbito privado fundamentalmente (Cubillos, 2019; Sapelle, 1990, pp. 257-279).

Durante la dictadura que se orientó hacia reformas neoliberales, sin contrapesos políticos, la flexibilización se configuró particularmente a partir de la crítica a la protección de los contratos contra despidos arbitrarios existentes desde 1966 (LEY 16455). Pocos días después del golpe de Estado de septiembre de 1973, el nuevo régimen buscó disminuir la protección a los contratos, aprovechando las condiciones de "excepcionalidad" y de fuerza que caracterizaron a la dictadura. La argumentación del régimen se orientaba a que la norma de protección permitía, tanto la existencia de una "politización" de los puestos de trabajo, la existencia de "agitadores políticos"

9. Citado por Rojas (2009, p. 6). Prat Echaurren, al examinar las 32 cajas previsionales y los 100 regímenes previsionales que demandaban una tasa de cotización equivalente al 61,9\% del sueldo imponible, constató el complejo escenario para el Estado. 
al servicio del proyecto socialista de la Unidad Popular, como a la afectación a los costos del trabajo. La junta usó el Decreto Ley 198 para eliminar militantes políticos en su primera fase. La política de austeridad en el gasto fiscal y rigidez en los salarios para los sectores públicos y privados se evidenció en dos ámbitos de la población activa en el bienio 1973-74: el despido de más de 100.000 funcionarios públicos y el éxodo de profesionales universitarios, que de acuerdo a Conicyt, entre diciembre de 1973 y octubre de 1974, afectaba al 42\% del área de ingeniería y tecnología; 15,1\% de las ciencias exactas y matemáticas, 12,8\% de las ciencias médicas; al 21,6\% de las ciencias agropecuarias y al 28,6\% de las ciencias sociales (González, 2010, p. 222). Las desvinculaciones de profesionales universitarios, fueron principalmente por razones políticas.

Después de eliminar los elementos "corporativistas" de la junta (principalmente el grupo en la Fuerza Aérea cercano al General Leigh), el gobierno promulgó El Plan Laboral de 1979. En ese momento, el gobierno planteó que las nuevas relaciones laborales debían ser flexibilizadas en relación a las necesidades de los empresarios o el "mercado", usando un eufemismo habitual (Llanos, 2018; Winn, 2004, pp. 22-24, 31-38).

Con anterioridad a la dictadura, los argumentos "económicos" sobre el costo del trabajo en Chile, como argumento para una reducción las remuneraciones "exageradas" y la preservación de empleos e impulsar mayor inversión, fueron particularmente desplegados por los economistas chilenos educados en la Universidad de Chicago, los “Chicago Boys" (De Castro, 1969, p. 37).

La relevancia de la flexibilización como elemento de clase favorable a los intereses del empresariado, queda clara en la concepción que el Almirante Merino (segundo al mando en la Dictadura) tenía sobre el significado de los trabajadores y sus protecciones contractuales,

"nadie analiza la situación desde ese punto de vista: de que hay que buscar el modo de que el empresario pueda contratar más gente en tal forma, que al emplear más personas eso no le signifique, si en un momento no la necesita, repito, no le signifique una lacra o un costo tal en su producción que le haga imposible contratarla" (Sesión Junta Militar, Acta, n. 343, 1978, p. 5).

La dictadura fue incapaz de mejorar los índices de empleo, fracasando sus ideas sobre una relación entre flexibilización y reducción del desempleo. Esto contrasta con el "éxito" "macroeconómico" del "milagro económico chileno" (Petras y Vieux, 1990; Richards, 1997, pp. 139-159) en términos de contención de la inflación, con la implementación de políticas neoliberales. Un "éxito" que en términos sociales tuvo un costo de más del 40\% de la población viviendo en condiciones de pobreza, en 1989.

Durante el siglo XX, se operó un cambio en el mercado del empleo que, en relación a la fuerza laboral femenina, el país prosiguió con ciclos fluctuantes, pues a 
principios de siglo, en 1907 su participación fue de un 28,9\%, manteniéndose sobre el $20 \%$ en las décadas de 1950-1960 y disminuyendo en 1970 a un 19,7\%, para repuntar en 1980 con un 22,30\% (Pardo, 1988, p. 33) ${ }^{10}$. No obstante, detrás de este indicador, se escondía una realidad que modificó la visión de la mujer chilena anterior a 1973, al enfrentar en dictadura tanto el trabajo informal y el exilio, este último con una dimensión de género (Gil, 2010, pp. 854-858; Rebolledo, 2009, pp. 537-545).

En relación a la incorporación de las mujeres al trabajo remunerado en Latinoamérica, desde la década de 1960, las transformaciones demográfico-educacionales impulsaron una mayor presencia de la mujer en empleos más diversos y a partir de la crisis de la deuda en 1982, esta incorporación se hizo más estable, configurándose muchos hogares con dos adultos trabajando. En esto habría tenido un rol causal importante la caída de salarios reales y el alto desempleo de los hombres (León, 2000, p. 15). Aun con estos cambios, las mujeres han mantenido la doble tarea del trabajo "remunerado" y las actividades domésticas ${ }^{11}$. En el último período, la participación de las mujeres en el trabajo asalariado en Chile ha seguido este patrón, pero aún su nivel es mucho menor que países con similares niveles de desarrollo socioeconómico, como Argentina y Uruguay. Además, las mujeres solteras sin hijos tienen una participación laboral mayor que las madres solteras y las madres casadas. Las mujeres universitarias tienen una mayor participación laboral que las mujeres con menos educación. Por último, la brecha salarial por género se ha reducido durante las últimas décadas, pero sigue vigente (Contreras et al., 2006, p. 99-146; PNUD, 2010, pp. 38, 114-133; 135-145; PNUD, 2017, p. 257-271).

La flexibilización del trabajo, se ha relacionado con el empleo femenino precario, muchas veces marginado de seguridades sociales, reducido en horas, etc. Para el caso de Chile, vemos que entre 1982 y 1992, el sector que experimentó el mayor crecimiento en participación femenina fue la agricultura que pasó del 0,7\% al 2,8\% respectivamente, mientras su participación en la industria manufacturera cayó del $21 \%$ al $16,4 \%$ y en la construcción disminuyó del 1,4 al 0,5\%. La rama económica donde se mantuvo predominante fue los servicios comerciales, sociales y personales, con índices de 44,1 a 42,7 respectivamente para el periodo señalado (Bank, 2017, pp. 108-133; Godoy et al., 2012, pp. 253-266; Martínez y Díaz, 1996, p. 105; Schurman, 2004, pp. 298-336; Tinsman, 2014, pp. 207-254; Valenzuela, 2012, pp. 149-162). Una parte de los servicios personales, fue el trabajo doméstico para otras familias, al que

10. La cifra de 1970 la discute Stiepovich y refiere un porcentaje de un 24,1\% y agrega para 1985 un porcentaje de 29,8\% (Stiepovich, 1998, p. 13).

11. Szasz (1994), observa que "Los mayores aumentos en la actividad económica femenina se registraron entre 1982 y 1984 . Esos años fueron los de más alto crecimiento de la desocupación masculina y de mayor disminución de los salarios" (Szasz, 1994, p. 22). 
muchas mujeres se dedicaron desde los años 1980, y que podría considerarse como una de las más radicales expresiones de flexibilización laboral, a favor del empleador: marginadas de los beneficios sociales, como el derecho maternal, con limitaciones en las indemnizaciones por despido, otro tratamiento en términos de horas de trabajo, etc. Esta dimensión del trabajo de mujeres de los sectores populares fue un importante factor para la acumulación de las familias de sectores más acomodados.

\section{Conclusión}

Las décadas de 1970 y 1980, fueron el marco temporal de un conjunto de crisis y transformaciones del capitalismo, que agotaron o impusieron límites al desarrollo de formas de Estado, que, aun manteniendo las relaciones capitalistas, habían impulsado importantes niveles de intervención política en la economía, en pos de mejorar las condiciones de bienestar social. Estas políticas, comunes en gran parte del occidente estuvieron ligadas a las propuestas keynesianas, con énfasis en la relevancia tanto del crecimiento productivo, como de la demanda de empleo. A estos rasgos se añadió el aumento de los niveles de organización en la influencia de los trabajadores en las discusiones políticas y económicas. En países centrales del capitalismo, como Alemania Federal, estos procesos estuvieron ligados al progresivo predominio político de la socialdemocracia, desde los años 1960. En Chile, un país semi-periférico, las propuestas críticas al capitalismo y de izquierda, también se fortalecieron desde los años 1960, asumiendo características más radicalizadas en la "vía chilena al socialismo".

Las dos crisis del petróleo (1973 y 1979) y la crisis de la deuda (1982) y sus efectos en la economía, fueron el marco para que las propuestas neoliberales (en desarrollo desde fines de la década de 1930) ganaran impulso dentro de las discusiones políticas, entregando las herramientas ideológicas y técnicas para modificar las relaciones entre el Estado y la economía.

A partir de los años 1970, Alemania Federal, experimentó un progresivo agotamiento del "milagro económico", que significó problemas a nivel de la demanda de empleo, se comenzó a registrar, en efecto un importante número de desempleados. A diferencia de lo ocurrido en otros países, el movimiento sindical alemán logró reducir el impacto de la crisis, vía la negociación de aumentos salariales moderados, reducción de las horas trabajadas por semana, y reducción de la fuerza laboral a través de las jubilaciones en vez que los despidos. Además, las formas de administración y negociación a nivel de las empresas, junto a la implementación de mejoras en la capacitación laboral, permitieron que la industria alemana mantuviera un buen nivel competitivo internacional, basado en la calidad. El hecho que tanto el partido socialdemócrata como el partido demócrata cristiano representaron distintos segmentos de los sindicatos significó que esas organizaciones recibirían algún tipo de apoyo bajo gobiernos de distintos “colores.” Este hecho, combinado con la tradición corporativis- 
ta de negociación colectiva y la popularidad de las políticas de bienestar entre toda la población lograron "frenar" los impulsos más radicalizados neoliberales en Alemania, provocando un proceso lento de liberalización (Prasad, 2006; Thelen, 2012; 2014).

En Chile, el impacto de la crisis internacional, particularmente observable desde 1974 se combinó con el establecimiento de la dictadura militar desde septiembre de 1973. La dictadura contó con amplios márgenes de poder para implementar medidas de shock anti-inflacionario, la reducción del número de funcionarios públicos, y relajación de las protecciones laborales. Las características del régimen implicaron que el movimiento sindical fuera incapaz de limitar o contener las transformaciones estructurales llevadas adelante por la dictadura. Las medidas de la dictadura de reducir el costo del trabajo y la inflación fracasaron rotundamente a la hora de mejorar la demanda de trabajo.

Otro fenómeno presente desde los años 1970 y 1980 fue la creciente importancia de la flexibilización laboral. Esta se desplegó en dos relevantes formas, primero en el trabajo "femenino" y segundo en el subcontrato, o contrato atípico. Estos fueron presentados como formas de adaptar la demanda de empleo a las condiciones económicas cada vez más competitivas e inciertas. Adaptaciones que han implicado una reducción de las "seguridades" laborales, en favor de las necesidades de los empleadores.

En Alemania, la flexibilización se relacionó con la falta de acceso a trabajos de tiempo completo de las mujeres y jóvenes. Esto encuentra una explicación en que el "modelo alemán" tendió a reforzar nociones económicas centradas en "el hombre proveedor" y un Estado que no desarrolla servicios adecuados de sala cuna que inhibe la participación laboral de las mujeres y desincentiva la fecundidad. En este marco se generó un mercado laboral segmentado donde los hombres tenían trabajos estables y las mujeres y jóvenes se integraban en posiciones más precarias. Aun así, el periodo $1970-1980$, no fue marco de reducciones drásticas en ayudas o prestaciones sociales, lo cual es una diferencia relevante en comparación con las lógicas de implementación radical del neoliberalismo, que como en Chile, precarizaba el empleo y los contratos, permitiendo que la libertad del mercado operara sin mayores contrapesos.

En línea con lo anterior, en el primer período de la dictadura (1973-1978), la flexibilización en Chile, operó con un conjunto de disposiciones sui generis que cuestionaron las regulaciones y protecciones de contratos, derechos laborales y despidos. En su período de la institucionalización (1978-1982), estas medidas, fueron sistematizadas, con normativas que posibilitaron los despidos de trabajadores sin especificación de causa.

En lo que respecta a la flexibilización y el trabajo femenino, los sectores más precarizados fueron los que aumentaron su participación en el empleo (trabajo agrícola y servicios). Capítulo importante en este ámbito de precarización y flexibilización es el 
del trabajo doméstico para otras familias, pues éste constituye una de las formas más radicales de flexibilización laboral, a favor del empleador, incluido el factor de incremento de acumulación de las familias de los sectores más acomodados. Hasta entrado el siglo XXI, estas trabajadoras estuvieron marginadas de los beneficios sociales, como el derecho maternal, con limitaciones a las horas de trabajo, remuneraciones inferiores al salario mínimo, sin derecho a indemnización por despido.

En las trayectorias de respuestas políticas y económicas frente a los efectos de las crisis de la década de 1970 y 1982, es relevante considerar las características de los regímenes políticos, los grados de democratización política y social, a lo que se debe añadir la profundidad e importancia histórica de la relación del Estado, tanto con la mantención de asignaciones de bienestar, como con el grado de influencia y participación de las organizaciones de trabajadores. En el caso de Alemania Federal, las características de participación multipartidista, como el nivel de importancia de los sindicatos, son factores explicativos relevantes a la hora de entender las formas en que el desempleo y la flexibilización se desenvolvieron. En ambos casos, los procesos de transformación o reforma fueron distantes de la radicalidad neoliberal que se observa en Chile durante la dictadura.

En Chile, las políticas implementadas por la dictadura tanto en relación al desempleo como a la flexibilización evidenciaron tempranamente procesos de precarización y de desplazamiento de la centralidad del empleo, hacia posturas marcadamente monetaristas y neoliberales, que contaron con los amplios márgenes de aplicación que la falta de democracia y participación de los trabajadores podía asegurar al proyecto neoliberal. De todas formas, durante los primeros años del régimen, las orientaciones neoliberales se fueron desplegando en relación a medidas sui generis que durante la segunda mitad de los años 1970 asumieron mayor coherencia y sistematización neoliberal. En este sentido, es posible observar que el Chile de los años 1980 en adelante, con una completa institucionalidad impuesta del modelo neoliberal se transformó en referencia de implementación neoliberal en muchos países democráticos.

Estos dos casos pueden interpretarse dentro de un contexto más amplio de respuestas nacionales a las transformaciones económicas globales y el auge ideológico del neoliberalismo a partir de los años 70. Representan dos "variedades de liberalización" (Thelen, 2014) que reflejan sus distintas trayectorias políticas y sistemas institucionales de relaciones industriales. El caso chileno representa un camino hacia una economía liberal de mercado que resulta de una polarización política y social que catalizó una alianza entre economistas, ideólogos neoliberales, grandes empresas, grupos políticos, y militares en ascenso que posteriormente fue seguido (bajo regímenes democráticos) por Inglaterra y los EEUU (Fourcade-Gourinchas y Babb 2002; Harvey, 2005; Prasad, 2006). Alemania representa otro camino de una economía coordinada de mercado en el cual los sindicatos con el apoyo de sectores de la democracia 
cristiana lograron "frenar" la implementación de políticas neoliberales radicalizados. La defensa de los trabajos manufactureros, sin embargo, provocó un mercado laboral segmentado en el cual creció la cesantía entre las mujeres y los jóvenes. Este camino alemán es similar al patrón de liberalización en Italia, Francia, y Holanda, con algunas variaciones (Thelen, 2014).

Este ejercicio de comparación entre un país central democrático con un sistema de economía coordinada de mercado con un país semi-periférico con una economía liberal de mercado amplia las posibilidades de sistematizar nuestro conocimiento comparado sobre las causas y consecuencias de las políticas de liberalización en distintas regiones del mundo. Este tipo de análisis se hace cada vez más importante porque en las décadas posteriores a las estudiadas en este artículo, las ideas neoliberales ya han influido no solamente a los partidos de derecha, sino también a los partidos de izquierda, tanto en Alemania, Chile, y muchos otros países (Huneeus 2001; Mudge 2018; Winn 2004).

Los casos de Chile y Alemania Federal, nos acercan a una mejor comprensión de las formas en que la flexibilización se ha configurado como un fenómeno que aún con sus diversas intensidades, es parte de un marco global común, en el horizonte de la historia y los estudios sobre el trabajo.

\section{Referencias}

Archivo Nacional. Ministerio del Trabajo Chile (1975). Vol. 3164, oficios 1-223.

Baccaro, Lucio y Chiara Benassi (2017). Throwing out the Ballast: Growth Models and the Liberalization of German Industrial Relations. Socio-Economic Review, 15 (1): 85-115.

Bank Muñoz, Carolina (2017). Building Power from Below: Chilean Workers Take on Walmart. Cornell University Press.

Bauer, Reinhold (2008). «Ölpreiskrisen und Industrieroboter». En K. H., Jarausch (Hrsg), Das Ende der Zurversicht? Die siebziger Jahre als Geschichte (pp. 68-83), Vandenhoehoeck \& Ruprecht.

Beaud, Michel (2012). Historia del capitalismo. Ariel.

Beck, Ulrich y Elisabeth Beck-Gernsheim (1994). Riskante Freiheiten: Individualisierung in modernen Gesellschaften. Suhrkamp Verlag.

Biglaiser, Glen (1999). Military Regimes, Neoliberal Restructuring, and Economic Development: Reassessing the Chilean Case. Studies in Comparative International Development, Spring, 3-26. 
Blossfeld, Hans-Peter, Dirk Hofäcker, Heather Hofmeister y Karin Kurz (2008). Globalisierung, Flexibilisierung und der Wandel von Lebensläufen in modernen Gesellschaften. En M. Szydlik (Hrsg), Flexibilisierung. Folgen für Arbeit und Familie (pp. 23-46), VS Verlag für Sozialwissenschaften, Wiesbaden.

Contreras, Dante, Esteban Puentes y Tomas Rau (2006). Apertura comercial y mujer trabajadora: el caso de Chile. En C. Piras (ed.), Mujeres y trabajo en América Latina: Desafíos para las políticas laborales (pp. 99 -146), Banco Interamericano de Desarrollo.

Cubillos, Paula (2019). L'Etat social au Chili sous le prisme des politiques de l'infance: continuités et inflexions 1973-2013 (Thése de doctorat de Sociologie).Université Paris Descartes. Recuperado de http://www.theses.fr/2017USPCB183.

Daher, Antonio (2013). El sector inmobiliario y las crisis económicas. Eure, 39 (118), 47-76.

De Castro, Sergio (1969). Política de Precios. Cuadernos de Economía, 6 (17): 34-40.

Der Spiegel (1973). Mit knappen Vorräten sorglos geaast, 47, p. 25.

Deutscher, Bundestag (1973). Wahlperiode - 67, Sitzung, Bonn, Donnerstag, 29 de Noviembre de 1973.

Diario de Sesiones del Congreso nacional. Actas Junta de Gobierno 1973-1990. Acta, n. 343. 1978. Recuperado de https://www.bcn.cl/historiapolitica/corporaciones/ periodos_legislativos?periodo=1973-1990.

Drake, Paul (1996). Labor Movements and Dictatorships: The Southern Cone in Comparative Perspective. Johns Hopkins University Press.

Drake, Paul (2003). El movimiento obrero en Chile. De la Unidad Popular a la Concertación. Revista de Ciencia Política, XXIII (2): 148-158.

Esping-Anderson, Gøsta (1996). Welfare States without Work: The Impasse of Labour Shedding and Familialism in Continental European Social Policy. En G. Esping-Anderson (ed). Welfare States in Transition: National Adaptations in Global Economies (pp. $72-84$ ), Sage.

Fillipi, Pablo, José Román, José y José Miguel Villena (2017). Estudios económicos estadísticos. Balance del Banco Central de Chile, 1926 a 2015. Banco Central de Chile.

Fischer, Karin (2009). The Influence of Neoliberals in Chile Before, During, and After Pinochet. En Ph. Mirowski, y D. Plehwe, (eds). The Road from Mont Pèlerin: The Making of the Neoliberal Thought Collective (pp. 305-346), Harvard University Press.

Fontaine, Arturo (1988). Los economistas y el presidente Pinochet. Zig-Zag.

Fourcade-Gourinchas, Marion y Sarah Babb (2002). The Rebirth of the Liberal Creed: Paths to Neoliberalism in Four Countries. American Journal of Sociology, 108 (3): 533-579. 
Gil, Fernanda (2010). Pensando el Sur. Uruguay, Chile y Argentina: mujeres, dictaduras y represión. En Ch. Fauré,. (dir.), Diccionario Akal. Enciclopedia histórica y política de las mujeres Europa y América (pp. 854-858), Ediciones.

Godoy, Lorena, Antonio Stecher y Juan Pablo Toro (2012). Acceso a derechos, sentidos de pertenencia y formas de acción colectiva en el trabajo: Una reflexión sobre el ejercicio de la ciudadanía en trabajadores de supermercados en Santiago de Chile. En A. Cárdenas, F. Link y J. Stillerman(eds), ¿Qué significa el trabajo hoy? Cambios y continuidades en una sociedad global (pp. 253-266), Catalonia.

Torres, Eugenio y Antonio González (1993). La política de bienestar social en los países de la OCDE hasta los años 90. Un balance. Cuadernos de Relaciones Laborales, 3, 277-297.

González, José (2010). Política, sociedad y relaciones internacionales en el último cuarto del siglo XX. En J. González, (Coord), Cincuenta años de historia de Chile vistos desde la revista Mensaje, II (pp 197-307), Ediciones Universitarias, Universidad Católica del Norte.

Hachette, Dominique (2000). Privatizaciones: Reforma estructural pero inconclusa. En F. Larraín y R. Vergara. La transformación económica de Chile (pp. 111-153). CEP.

Hall, Peter y Kathleen Thelen (2009). Institutional Change in Varieties of Capitalism. Socio-Economic Review, 7, 7-34.

Harvey, David (2005). A Brief History of Neoliberalism. Oxford University Press.

Hielscher, Volker (2000). Entgrenzung von Arbeit und Leben? Die Flexibilisierung von Arbeitszeiten und ihre Folgewirkungen für Beschäftigten. Eine Literaturstudie. WZB Discussion Paper, N. FS II 00-201. Wissenschaftszentrum Berlin für Sozialforschung.

Huneeus, Carlos (2001). El gobierno de Pinochet. Editorial Sudamericana.

Kenworthy, Lane (2002). Corporatism and Unemployment in the 1980s and 1990s. American Sociological Review, 67 (3): 367-388.

Kramer, Nicole (2012). Neue Soziale Bewegungen, Sozialwissenschaften und die Erweiterung des Sozialstaats. Familien - und Altenpolitik in den 1970er und 1908er Jahren. Archiv für Sozialgeschite 52 (pp. 211-230). Bonn.

Layard, Richard, Stephen Nickell y Richard Jackman (2003). Unemployment. Macroeconomic Performance and the Labour Market. Oxford University Press.

León, Francisco (2000). Mujer y trabajo en las reformas estructurales latinoamericanas durante las décadas de 1980 y 1990. CEPAL.

Lessenich, Stephan (2017). Neben uns die Sinflut. Hanser Berlin. 
Lindner, Stephan (2008). Die westdeutsche Textilindustrie zwischen Wirtschaftswunder und Erdölkrise. En K. H. Jarausch (Hrsg) Das Ende der Zurversicht? Die siebziger Jahre als Geschichte (pp. 49 - 67). Vandenhoehoeck \& Ruprecht.

Llanos, Claudio (2018). La dictadura militar en Chile frente al desempleo: algunos aspectos de la mirada política 1973-1978. Estudos Ibero-Americanos, 44 (2): 311325.

Martínez, Javier y Álvaro Díaz (1996). Chile: The Great Transformation. Brookings Institution.

Möller, Joachim (2015). Did the German Model Survive the Labor Market Reforms?. Journal of Labor Market Research, 48, 151-168.

Mudge, Stephanie (2018). Leftism Reinvented: Western Parties from Socialism to Neoliberalism. Harvard University Press.

Muiños, Benito (1999). Neoliberalismo y desigualdad en Europa y América". Scripta Nova. Revista Electrónica de Geografía y Ciencias Sociales, 45(9).

Nullmeier, Frank y Franz Xaver Kaufman (2010). Post-War Welfare Sate Development. En F. Castles, et. al (edit), The Oxford Handbook of the Welfare State (pp. 81 - 101). Oxford University Press.

OCDE. (2019). El gasto público social es alto en muchos países de la OCDE. Social Expediture Update 2019.

Offe, Claus (1990). Contradicciones en el Estado del Bienestar. Alianza Universidad.

Organización Internacional del Trabajo (OIT) (1998). Repercusión de las disposiciones sobre flexibilidad de los mercados de trabajo en las industrias mecánicas, eléctricas y electrónicas. Informe para el debate de la Reunión Tripartita sobre la Repercusión de las disposiciones sobre flexibilidad de los mercados de trabajo en las industrias mecánicas, eléctricas y electrónicas. Oficina Internacional del Trabajo: Recuperado de https://www.ilo.org/global/about-the-ilo/newsroom/news/ WCMS_008509/lang--es/index.htm.

Oschmiansky, Heidi y Frank Oschmiansky (2003). Erwerbsformen im Wandel: Integration oder Ausgrenzung durch atypische Beschäftigung: Berlin und die Bundesrepublik Deutschland im Vergleich. Discussion Papers. Wissenschaftszentrum Berlin für Sozialforschung. GmbH. Recuperado dehttps://nbn-resolving.org/ urn:nbn:de:0168-ssoar-11171.

Pardo, Lucía (1988). Una revisión histórica de la participación de la población en la fuerza de trabajo. Tendencias y características de la participación de la mujer. $R e$ vista de Economía, 15 (1): 25-82.

Petras, James y Steve Vieux (1990). The Chilean "Economic Miracle": An Empirical Critique. Critical Sociology, 17 (2): 57-72. 
Piketty, Thomas (2014). El capital en el siglo XXI. Fondo de Cultura Económica.

PNUD (2010). Desarrollo Humano en Chile 2010. Género: Los desafíos de la igualdad. Programa de las Naciones Unidas de Desarrollo.

PNUD (2017). Desiguales. Orígenes, cambios y desafíos de la brecha social en Chile. Programa de las Naciones Unidas para el Desarrollo.

Prasad, Monica (2006). The Politics of Free Markets: The Rise of Neoliberal Economic Policies in Britain, France, Germany \& the United States. University of Chicago Press.

Rapoport, Mario y Noemí Brenta (2010). La crisis económica mundial:¿El desenlace de cuarenta años de inestabilidad?. Problemas del Desarrollo, 41(163): 7-30.

Rebolledo, Loreto (2009). Chilenas en el exilio. En Montecino, Sonio (Compiladora), Mujeres chilenas fragmentos de una historia (pp. 537-545), Editorial Catalonia.

Richards, Donald (1997). The Political Economy of the Chilean Miracle. Latin American Research Review, 32 (1): 139-159.

Riveros, Luis (1984). Un análisis sobre el problema del empleo en Chile en la década del 70. Estudios de Economía, Segundo Semestre, 1-28.

Rojas, Alejandro (2009). Reforma al Sistema Previsional. Memoria para optar al Título Profesional de Licenciado en Ciencias Jurídicas y Sociales. Universidad de Chile.

Rosende, Francisco (1988). Una interpretación del desempleo en Chile. Estudios Públicos 32, 67-128.

Sapelli, Claudio (1990). Ajuste estructural y mercado de trabajo. Una explicación de la persistencia del desempleo en Chile: 1975-1980. Estudios de Economía, 17 (2): 257-279.

Sassoon, Donald (2001). Cien años de socialismo. Edhasa.

Schurman, Rachel (2004). Shuckers, Sorters, and Gutters: Labor in the Fisheries Sector. En P. Winn. (ed.). Victims of the Chilean Miracle: Workers and Neoliberalism in the Pinochet Era, 1973-2002 (pp. 298-336). Duke University Press.

Schwartz, Michael (2008). Frauen und Reformen im doppelten Deutschland. Zusammenhänge zwischen Frauenerwerbsarbeit, Abtreibungsrecht und Bevölverungspolitik um 1970. En K. H. Jarausch, (Hrsg). Das Ender der Zurversicht? (pp. 196 - 214). Vandenhoeck \& Ruprecht.

Spitznagel, Eugeny Susanne Wanger. (2004). Arbeitszeitpolitik: Mit längeren Arbeitszeiten aus der Beschäftigungskrise? IAB-Kurzbericht, 10. Recuperado de https:// www.econstor.eu/handle/10419/158172.

Stiepovich, Jasna (1998). Fuerza laboral de la mujer en Chile: cifras y características. Revista Latino-Americana de Enfermagem, 6, 53-58. 
Streeck, Wolfgang (1997). German Capitalism: Does it Exist? Can it Survive?. New Political Economy, 2 (2): 237-256.

Süß, Dietmar y Meik Woyke (2012). Schimanskis Jahrzehnt? Die 1980er Jahre in historischer perspektive. Archiv für Sozialgeschichte, 52. (pp. 3 - 20). Bonn.

Süß, Dietmar (2012). Stempel, Stechen, Zeit erfassen.Überlegugen zu einer Ideen und Sozialgeschiche der 'Flexibilisierung'. Archiv für Sozialgeschichte. Band 52. Bonn. Verlag J.H.W. Dietz Nachf.

Szasz, Ivonne (1994). La mujer en el trabajo y la migración. El mercado laboral femenino entre 1950 y 1990 y la inmigración de mujeres a la ciudad de Santiago de Chile. Notas de Población, 59, 9-50.

Thelen, Kathleen (2012). Varieties of Capitalism: Trajectories of Liberalization and the New Politics of Social Solidarity. Annual Review of Political Science, 15, 137159.

Thelen, Kathleen (2014). Varieties of Liberalization and the New Politics of Social Solidarity. Cambridge University Press.

Tinsman, Heidi (2014). Buying into the Regime: Grapes and Consumption in Cold War Chile and the United States. Duke University Press.

Valenzuela, María Elena y Solange Sanches (2012). Trabajo doméstico e identidad: Las trabajadoras domésticas remuneradas en Chile. En A. Cárdenas, F. Link y J. Stillerman(eds.) ¿Qué significa el trabajo hoy? Cambios y continuidades en una sociedad global (pp. 149 - 162), Catalonia.

Vergara, Pilar (1984). Auge y caída del Neoliberalismo en Chile. FLACSO, Documento de Trabajo $\mathrm{N}^{\circ} 126$.

Werding, Martin (2008). Gab es eine neoliberale Wende?. Vierteljahrshefte für Zeitgeschichte, 56 (2), 303-321.

Winn, Peter (2004). The Pinochet Era. En P. Winn, (ed.). Victims of the Chilean Miracle: Workers and Neoliberalism in the Pinochet Era, 1973-2002 (pp. 14-70). Duke University Press. 


\section{Sobre los autores}

Claudio Llanos R. es Profesor de Historia Contemporánea en el Instituto de Historia de la Pontificia Universidad Católica de Valparaíso, Chile. Doctor por la Universidad de Barcelona, España. Fellow de la Fundación Alexander von Humboldt y del Centro de Estudios Avanzados de la Universidad de Múnich. Líneas de investigación centradas en las relaciones Estado y Sociedad, políticas de bienestar, trabajo y desempleo.Correo Electrónico: claudio.llanos@pucv.cl. iD https://orcid.org/0000-0002-76125497

José Antonio González P. es Académico-Investigador, Profesor Titular de la Facultad de Ciencias Jurídicas, Universidad Católica del Norte. Doctor en Historia por la Universidad de Navarra. Autor de libros y de artículos sobre la industria salitrera, literatura e historia de Chile, relaciones internacionales latinoamericanas y el paisaje del desierto de Atacama y la cultura minera. Correo Electrónico: jagonzal@ucn.cl.

https://orcid.org/0000-0002-4030-0353

Joel Stillerman es Doctor en Sociología con mención en Estudios Históricos por la New School for Social Research. Profesor Titular de Sociología en Grand Valley State University. Autor de The Sociology of Consumption: Global Approach (2015, Polity) y co-editor de ¿Qué significa el trabajo hoy? (2012, Catalonia). Autor de varios artículos y capítulos sobre el movimiento obrero, la sociología del consumo, y las clases medias en Chile, América Latina, y el Sur Global. Department of Sociology, Grand Valley State University, USA. Correo Electrónico: stillejo@gvsu.edu. 


\title{
CUHSO
}

Fundada en 1984, la revista CUHSO es una de las publicaciones periódicas más antiguas en ciencias sociales y humanidades del sur de Chile. Con una periodicidad semestral, recibe todo el año trabajos inéditos de las distintas disciplinas de las ciencias sociales y las humanidades especializadas en el estudio y comprensión de la diversidad sociocultural, especialmente de las sociedades latinoamericanas y sus tensiones producto de la herencia colonial, la modernidad y la globalización. En este sentido, la revista valora tanto el rigor como la pluralidad teórica, epistemológica y metodológica de los trabajos.

\author{
EDITOR \\ Matthias Gloël \\ COORDINADORA EDITORIAL \\ Claudia Campos Letelier \\ CORRECTOR DE ESTILO Y DISEÑADOR \\ Ediciones Silsag \\ TRADUCTOR, CORRECTOR LENGUA INGLESA \\ Aurora Sambolin Santiago \\ SITIO WEB \\ cuhso.uct.cl \\ E-MAIL \\ cuhso@uct.cl \\ LICENCIA DE ESTE ARTÍCULO \\ Creative Commons Atribución Compartir Igual 4.0 Internacional
}

